\title{
Fernández Caso, M. V.; Gurevich, R. (dirs.) (2014). Didáctica de la Geografía. Prácticas escolares y formación de profesores.
}

Buenos Aires: Biblos, 309 pp.

OSCAR LOSSIO

Facultad de Humanidades y Ciencias, Universidad Nacional del Litoral, Santa

Fe, Argentina.

olossio@hotmail.com

En la Argentina, y en coincidencia con lo que sucede en la mayoría de los países de América Latina, se observa una menor producción en investigación en la Didáctica Especial frente a otras áreas de especialización del quehacer académico y profesional de la Geografía. Si bien podemos reconocer que la indagación relativa a la enseñanza disciplinar se ha acrecentado notablemente en cantidad y en calidad en los últimos años, lo que se puede observar tanto en las comunicaciones en distintos eventos académicos como en publicaciones en revistas especializadas, sigue siendo escasa la edición de libros sobre la temática y más aún de aquella que refiera a la investigación. Precisamente, si se le puede reconocer una gran virtud a esta compilación, a cargo de María Victoria Fernández Caso y Raquel Gurevich, es que permite una mayor visibilidad de la producción de distintos equipos de trabajo de algunos países de América Latina, a partir de la inclusión de textos que socializan investigaciones ya finalizadas o que están en proceso, y de otros artículos de reflexión que, en su mayoría, citan algunos resultados de indagaciones ya desarrolladas previamente por sus autores.

Es necesario señalar que la obra presenta trabajos que reflejan distintos abordajes metodológicos, adscripciones teóricas, grados de avance en el desarrollo de las investigaciones que se presentan, estilos de escritura y diferentes formas de organización. De igual modo, la diversidad también se evidencia en cuanto al modo en que se vinculan con los proyectos de investigación de los equipos autorales y lo que cada uno de ellos ha seleccionado para socializar; por ejemplo, algunos textos sólo ofrecen abordajes conceptuales y no exhiben ni los resultados ni la perspectiva metodológica elegida. Sin embargo, consideramos necesario subrayar 
que gran parte de las producciones dan cuenta de la renovación teórica y metodológica que se viene desarrollando en la Geografía en general y en la Didáctica Especial en particular. En cuanto a lo metodológico, destacamos en espacial algunos artículos que exponen interesantes ejemplos de investigaciones de carácter participativas que incluyeron instancias de desarrollo profesional docente, lo que les ha permitido focalizarse en el análisis de la reflexión de los docentes sobre sus prácticas, sobre cómo problematizan la enseñanza y sobre cómo piensan y actúan en torno a la innovación en la enseñanza. A su vez, coincidimos con las compiladoras cuando expresan que las propuestas de los autores se distinguen por repensar teorías, identificar problemas y proponer hipótesis, y por plantear nuevas interpretaciones acerca de los procesos de aprender y enseñar geografía en distintos niveles educativos. Por todo lo señalado precedentemente, consideramos que el libro es un valioso aporte para las cátedras de Didáctica Especial y otras de la formación docente en la especialidad, y es de utilidad tanto para la docencia de grado y de posgrado, como para los equipos de investigación, los tesistas y para otros interesados en iniciarse en la indagación sobre la enseñanza de la geografía.

El libro se editó en el marco de la participación de los autores en el III Coloquio Internacional en Didáctica de la Geografía, correspondiente a la Red Latinoamericana de Investigadores en Didáctica de la Geografía (LADGEO), que se desarrolló en la Ciudad Autónoma de Buenos Aires en octubre de 20I4. Del total de dieciséis artículos, seis están escritos en portugués y corresponden a autores brasileros. De los diez que se presentan en español, siete son de autores argentinos, uno de chilenos, otro de colombianos y, uno en coautoría entre profesores de Brasil y de Colombia. La publicación se organiza en tres partes que, según las palabras de las compiladoras, condensan temáticamente los intereses y preocupaciones que se vienen compartiendo en el ámbito de la mencionada Red: I) Investigación didáctica y enseñanza de la geografía en América Latina; 2) Prácticas docentes y formación profesional en geografía, y 3 ) Saberes, experiencias y valoraciones de la geografía escolar. A continuación se realiza una breve descripción de cada texto.

La primera parte del libro inicia con el artículo de las directoras, titulado «Didáctica de la Geografía: Notas de investigación y problematización de las prácticas de enseñanza». En éste se presentan, a través de diferentes tópicos, aspectos que se vinculan con las opciones epistemológicas, políticas y pedagógicas de las autoras en sus prácticas profesio- 
nales que incluyen la formación docente inicial y continua, experiencias de investigación y prácticas de divulgación y de socialización. Sostienen que desde la Universidad buscan promover el mejoramiento de las prácticas docentes en Geografía, en el marco de las transformaciones de la enseńanza de la disciplina y la renovación del temario escolar. Señalan el rol estratégico que tiene la investigación en ese sentido, explicitando brevemente las premisas, los supuestos teóricos y la modalidad participativa de proyectos que han desarrollado, para acercarse a las formas en que los profesores conciben la enseñanza y la problematizan. Asimismo, valoran la creación de Redes colaborativas en Didáctica de la Geografía construidas en los últimos ańos como espacios de intercambio de producciones y comunicación de saberes, proyectos y experiencias. En el último apartado aportan algunas notas sobre cómo contribuir, desde la enseñanza de la disciplina, a la formación ciudadana y política de nuestros jóvenes.

El segundo artículo: «Enfoques geográficos, estrategias didácticas y formación ciudadana en Medellín (Colombia) y San Pablo (Brasil)» corresponde a Alberto Gutiérrez Tamayo, Raquel Pulgarín y Sônia María Vanzella Castellar. Trata sobre la potencialidad pedagógica de las categorías de: ciudad, de lo urbano, de lugar y de usos del suelo, para la formación ciudadana en la enseñanza disciplinar. El texto se ocupa de enmarcar teóricamente dichas categorías con aportes de la geografía crítica. En ese sentido, recuperamos un fragmento los autores: «Estudiar la ciudad, lo urbano, el lugar y los usos del suelo implica, entre otras cosas, asumirlos como espacios geográficos semantizados, usados, apropiados y construidos socioculturalmente, producto de las transformación histórica efectuada por los sujetos, como escenarios que favorecen o imposibilitan el ejercicio de las ciudadanías» ( $5 \mathrm{I})$. A partir de lo precedente, el trabajo presenta reflexiones sobre la enseñanza de la Geografía en relación con los procesos de formación ciudadana. Sin embargo, cabe notar que no se explicitan ni el enfoque metodológico ni resultados del estudio comparado que, según lo que sostienen los autores, estaba en ejecución al momento de presentar el escrito, por lo que consideramos que el título elegido no es estrictamente representativo del contenido del artículo.

El trabajo «Estudio del lugar y el libro didáctico en la enseñanza y el aprendizaje de la Geografía» corresponde a Helena Copetti Callai, quien señala la necesidad de brindar oportunidades a los jóvenes para que puedan acceder a un conocimiento que los empodere a partir de 
herramientas intelectuales que les permitan ejercer la ciudadanía y es por ello que le otorga un rol central en la enseñanza al trabajo a partir del concepto de lugar. Asimismo, reflexiona sobre cómo el lugar ha sido trabajado en la escuela y ha sido presentado en algunas propuestas curriculares. Además explicita algunos interrogantes en torno a cómo los libros didácticos presentan los contenidos de lugar, de ciudad y de lo urbano, y continúa con la reflexión sobre el papel de los libros de textos en la enseñanza, aunque no presenta resultados de la investigación.

El cuarto artículo «Materiales Curriculares para profesores de Geografía: contextos de producción y formas de uso", corresponde a Viviana Zenobi, y versa sobre la fundamentación teórica, la metodología y los resultados de investigaciones desarrolladas bajo su dirección en la Universidad Nacional del Luján (Argentina). Asimismo, presenta los objetivos con los que se elaboraron cuatro materiales curriculares, como parte de sus funciones en un equipo técnico de la Dirección Curricular del Ministerio de Educación de la Ciudad Autónoma de Buenos Aires, y describe brevemente cada uno de ellos. Como señala la autora, orientó sus investigaciones hacia la indagación de las prácticas de docentes que utilizaron esas producciones, con la finalidad de conocer los criterios puestos en juego en la selección de materiales educativos, comprender cómo estos influyen en el pensamiento del profesor y en sus decisiones didácticas y, además, para validar los criterios del equipo autoral que los elaboró. Consideramos de gran relevancia la manera en que se detalla y fundamenta el abordaje metodológico, lo que es acorde a la perspectiva interpretativa y al enfoque biográfico-narrativo. Además, sostiene que se encuadra en la corriente crítica porque focaliza en el compromiso explícito con lo ideológico, al tratar de comprender para transformar la práctica educativa con la participación de los protagonistas. Por eso también alude a la investigación-acción.

Andrea Coelho Lastória y Silvia Aparecida de Sousa Fernandes son las autoras del artículo "Avances y desafíos en la enseñanza de la Geografía en Brasil: formación de profesores y prácticas escolares». Presentan reflexiones en torno a qué concepciones de Geografía y de Geografía Escolar tienen los profesores brasileros, en articulación con sus trayectos formativos, presentando ejemplos hipotéticos sobre biografías escolares y de la formación inicial de quienes estudiaron en diferentes décadas. Al mismo tiempo, abordan cuál es el lugar de la Geografía en el currículum oficial de la escuela básica y en la formación de profesores para ese nivel. 
La segunda parte del libro, se inicia con el artículo de Fabián Araya Palacios y Yudi Herrera Núnez: "Aportes de la investigación en Didáctica de la geografía a la formación docente: los casos de República Dominicana y Chile», que exhibe la fundamentación teórica, la metodología y los resultados del proyecto: "Desarrollo del pensamiento espacial y comprensión sistémica del espacio geográfico como fundamento para la mejora integral de la educación geográfica, en un contexto de cooperación internacional», desarrollado entre la Universidad de La Serena (Chile) y la Universidad Autónoma de Santo Domingo. El problema planteado fue: ¿cuáles son las estrategias didácticas utilizadas por los docentes de la República Dominicana y Chile para desarrollar con sus alumnos el desarrollo espacial sistémico? El abordaje metodológico se limita a la aplicación de una encuesta a un grupo de docentes de Geografía de Educación básica (primaria) y secundaria de ambos países, y se presenta un análisis comparativo de sus resultados. Consideramos que el abordaje del problema sólo desde encuestas permite una aproximación limitada, por lo que sería interesante poder complementar la indagación con metodología cualitativa que permita una mayor compresión de los modos de pensar y de ejercer la enseñanza por parte de los docentes.

El siguiente artículo se denomina «El reto de enseñar problemas ambientales desde la complejidad: experiencias en el marco de un proyecto de investigación-acción en Buenos Aires» que corresponde a Andrea Ajón, Lia Bachmann y Patricia Souto. Lo destacamos por la solvente e interesante reflexión sobre el proceso metodológico utilizado. Mediante un trabajo de investigación participativa buscaron favorecer un entorno reflexivo junto a los profesores de escuelas secundarias, con los que interactuaron en talleres de producción de secuencias didácticas sobre problemáticas ambientales. Considerando al docente como un profesional reflexivo, las autoras sostienen que configuraron la investigación en instancias de planificación, acción, observación y reflexión, y subrayan que el trabajo realizado constituyó un espacio colectivo de producción. El texto también justifica la elección de la temática ambiental y presenta los principales resultados.

Luego, el trabajo "La formación docente en Colombia y su incidencia en la enseñanza de la Geografía», del Grupo Interinstitucional de Investigación Geopaideia, presenta, en primer lugar, un estado de situación de la enseñanza de la Geografía en Colombia, haciendo alusión a la 
evolución de ciertas normativas legales en las últimas cinco décadas. En segundo lugar, se analiza el lugar de la geografía en la formación del docente de Ciencias Sociales en el contexto universitario tomando las experiencias distintas de dos universidades. En tercer lugar, presentan sucintamente investigaciones que han desarrollado desde el Grupo Geopaideia; y, en la cuarta sección, presentan breves reflexiones sobre la estrategia de trabajo de campo y sobre el lugar del libro de texto en la enseñanza de la geografía.

Otro de los artículos que destacamos por su esmerada presentación de la metodología utilizada es de la autoría de Liliana Trigo, Analía Rotondaro, Natalia Flores y Andrés Flouch, y se denomina: «Reflexión y acción en torno a la interculturalidad en el pensamiento del profesores de Geografía que trabajan con alumnos de sectores populares de General Rodríguez, provincia de Buenos Aires». Se presentan conclusiones de un proyecto desarrollado entre 2009 y 2013 en la Universidad de Luján, que tuvo como objeto de análisis las representaciones que los profesores de Geografía de una escuela media tienen acerca de la diversidad cultural de sus alumnos y cómo la abordan en sus clases. Estudiaron si se modificaron las representaciones después de un proceso de discusión, del intercambio y de lectura de bibliografía en grupos, teniendo en cuenta los resultados de una investigación anterior. Para ello, implementaron grupos de discusión en los que introdujeron aportes teóricos para promover la reflexión fundamentada sobre las prácticas y realizaron análisis temático por etapas.

Para finalizar la segunda parte del libro, se incluyen, por un lado, el texto de Alejandra Marek, Jacqueline Dalinger y Alejandra Anahí Laiker, que se titula «Concepciones geográficas de profesores de Escuelas secundarias de Paraná, Entre Ríos» y se trata sólo del plan de un proyecto de investigación que, al momento de la publicación, no se había desarrollado; y por otro lado, el artículo «Formación de posgrado en didáctica de las ciencias sociales en la Universidad Nacional de la Patagonia San Juan Bosco" de Sergio Merino, presenta el programa de la Maestría en Didáctica Específicas de su Universidad, en el que se aborda principalmente sus propósitos, la estructura curricular y las orientaciones para la construcción de planes de tesis.

La tercera parte del libro, en la que predominan los artículos de autores brasileros, se inicia con el denominado "Formación básica, jóvenes ciudadanos y contenidos escolares: ¿qué lugar tiene la cultura 
de los alumnos en la enseñanza de la geografía?», de Lana de Souza Cavalcanti. La autora recupera aportes de dos investigaciones que viene desarrollando, la que son relativas a la formación del profesor, una de ellas vinculada a la educación ciudadana y la otra, a los jóvenes y la vida urbana cotidiana. Esencialmente, el texto incluye reflexiones y experiencias sobre las prácticas espaciales cotidianas de los jóvenes en la ciudad, con la base de testimonios de alumnos de enseñanza media y de ingresantes a carreras de Geografía en la Universidad. Entre otras cuestiones, toma en cuenta los lugares que se apropian, los espacios que valorizan por su calidad ambiental y los problemas urbanos que perciben, recogiendo demandas que se vinculan con las aspiraciones de los jóvenes, sobre lo que les gustaría tener y hacer, especialmente en los tiempos de ocio. Posteriormente, presenta algunas propuestas para la formación ciudadana en las clases de Geografía y hace mención a proyectos de intervención desarrollados desde la Universidad Federal de Goiás.

Sônia María Vanzella Castellar y Julio Cesar Epifânio Machado, en su artículo «La enseñanza de la geografía física en la educación básica: la superación del obstáculo para el aprendizaje», señalan la importancia de los conocimientos previos a partir de las nociones de opinión y de cultura experiencial, con las que abordan posibles dificultades para el aprendizaje de los alumnos, particularmente lo vinculado al problema de la opinión animista de los fenómenos de la materia. A su vez, exponen el enfoque del «Análisis geográfico integrado» a partir del que elaboraron secuencias didácticas, que utilizaron como instrumento de investigación, y explicitan los resultados de su aplicación.

Marcos Antônio Campos Couto, en su texto «Prácticas educativas en la geografía que se enseña en la escuela pública brasilera» comienza presentando diversos aspectos por los que considera problemática la educación pública brasilera. Continúa con la evolución histórica de la escuela en Brasil y lo vincula con la historia de la geografía y con algunos aspectos del rol de la disciplina en el currículo escolar. Además, de manera sucinta menciona algunas actividades pedagógicas realizadas en escuelas públicas por parte alumnos de nivel superior y en el marco de un proyecto institucional.

El artículo "Que saben y qué quieren saber los estudiantes secundarios sobre problemas ambientales» de Silvina Quintero, Mariana Saidón y Lia Bachmann, consigna un diagnóstico acerca de las representaciones de los alumnos sobre las problemáticas ambientales, sobre las expec- 
tativas y demandas que tienen hacia la escuela sobre el campo de lo ambiental y sobre qué vinculación establecen entre la Geografía y esta temática. El diagnóstico fue realizado mediante encuestas y se articula con la línea de investigación de un equipo que integran las autoras, que estudia la imagen pública de la Geografía.

El último artículo «Lugares escondidos, identidades silenciadas: ¿Territorios en red?», de Jerusa Vilhena de Moraes y Jorge Luis Barcellos da Silva, da cuenta de una experiencia de investigación desarrollada junto a estudiantes universitarios en el marco de una unidad curricular del curso de Pedagogía denominada Práctica Pedagógica Programada. Indagaron las relaciones entre escuela, familias, profesores y directores, focalizando en cómo repercute la localización de una escuela básica en el trabajo docente y en la vida de los alumnos y familias. De esta manera, señalan el carácter territorial de los procesos educativos en el barrio.

Luego de recorrer las distintas instancias de este importante y reciente aporte bibliográfico, integrado por distintas formas y recortes escriturales, es posible advertir que la obra podría haber tenido mayor consistencia si todos los trabajos hubiesen incluido la definición de la perspectiva metodológica y la presentación de resultados de cada investigación. Sin embargo, volvemos a remarcar nuestro saludo a esta publicación colaborativa que permite la difusión de la producción de investigaciones en el área de la Didáctica Especial, y esperamos que pueda continuarse con nuevas entregas que socialicen otros avances del trabajo de equipos de distintas universidades de América Latina. En ese sentido, la novedad editorial es una iniciativa muy bienvenida que respalda el camino de integración de equipos universitarios preocupados por el fortalecimiento del campo. 